\title{
Exploring Partially Confined Phases
}

\author{
Michael C. Ogilvie ${ }^{* \dagger}$ \\ Washington University \\ E-mail: mco@physics. wust l.edu
}

Peter N. Meisinger

Washington University

E-mail: pnmephysics.wust l.edu

\section{Joyce C. Myers}

Washington University

E-mail: jomyers@wust l.edu

Phases of $\mathrm{SU}(\mathrm{N})$ gauge theories in which the global $\mathrm{Z}(\mathrm{N})$ symmetry breaks spontaneously to a subgroup $\mathrm{Z}(\mathrm{L})$ can be realized by adding appropriate Wilson line terms to the gauge action. These phases are partially confining, in the sense that quarks are confined but bound states of $\mathrm{L}$ quarks are not. At temperatures large compared to the normal deconfinement temperature, the phase diagram, pressure, string tensions, and 't Hooft loop surface tensions can be calculated analytically. Approximate scaling laws emerge naturally for both string tensions and surface tensions.

The XXV International Symposium on Lattice Field Theory

July 30 - August 42007

Regensburg, Germany

\footnotetext{
*Speaker.

${ }^{\dagger} \mathrm{MCO}$ and JCM gratefully acknowledge the support of the U.S. Dept of Energy.
} 


\section{Introduction}

In recent work on $S U(3)$ and $S U(4)$ gauge theories, Myers and Ogilvie have shown that the addition of a term of the form $T \lambda_{A} T_{A} P$ to the gauge theory Lagrangian, where $P$ is the Polyakov loop, produces novel phases at temperatures above the pure gauge deconfinement transition temperature [1]. The new phase of $S U(4)$ is a partially confined phase, where the $Z(4)$ global symmetry spontaneously breaks to $Z(2)$, indicating confinement of quarks but not diquark states. The new phase of $S U(3)$ is distinct from the confined and deconfined phases, and occurs at intermediate values of $\lambda_{A}$. For large values of $\lambda_{A}$, the confined phase of $S U(3)$ is restored. In both $S U(3)$ and $S U(4)$, a theoretical analysis of the phase structure and thermodynamics is in good agreement with lattice simulation results. This has prompted us to a detailed analytical study of the general case of $S U(N)$. For a class of models with extended actions, we can study many new high-temperature phases, including confining and partially confining phases. We add to the gauge theory Lagrangian an external potential of the form

$$
V_{e x t}=\sum_{k=1}^{[N / 2]} \lambda_{k} \operatorname{Tr}_{F} P^{k} \operatorname{Tr}_{F} P^{\dagger k}
$$

which is both gauge-invariant and center-symmetric, i.e., invariant under global $Z(N)$ symmetry. We are able to calculate the phase structure, thermodynamics, string tensions, 't Hooft loop surface tensions, and Debye screening masses associated with all these phases at high temperatures

Our work is partially motivated by our previous work on an effective potential approach to the deconfinement transition [2-4]. Additional motivation comes from the work of Davies et al. [5, 6] on the role of monopoles in supersymmetric theories on $R^{3} \times S^{1}$, and of Diakonov et al. [7] on calorons in $S U(N)$ gauge theories at finite temperature. These works have demonstrated how topological excitations can make non-perturbative contributions to the effective potential that are crucial in determining phase structure and associated properties. In both cases, the leading contribution of the topological excitations to the effective potential is a term of the form $\lambda_{1} \operatorname{Tr}_{F} P \operatorname{Tr}_{F} P^{\dagger}$ with $\lambda_{1}$ positive. On the other hand, normal particles, including the gauge bosons themselves, make a negative contribution to $\lambda_{1}$ [8]. Generally, a negative value of $\lambda_{1}$ favors the $Z(N)$-breaking deconfined phase. However, positive $\lambda_{1}$ favors $\operatorname{Tr}_{F} P=0$, a defining property of the confined phase. For $N>3$, the additional terms in $V_{e x t}$ are needed to give confinement for all non-zero $N$-ality states.

\section{Form of the Effective Action}

The form of the effective potential for Polyakov loop eigenvalues is known from perturbation theory for weak coupling $[9,10]$, and should be valid for temperatures far above the deconfinement temperature of the pure gauge theory. This perturbative effective action gives us considerable information about high-temperature behavior, including the pressure and the Debye screening mass in the plasma. Kink solutions give analytical predictions for 't Hooft loop surface tensions $\rho_{k}$ $[11,12]$ that can be compared with lattice results $[13,14]$.

At the level of approximation at which we are working, the addition of $V_{\text {ext }}$ to the Lagrangian also adds it to the effective potential. We thus consider an effective action for Polyakov loops of 
the form

$$
S_{e f f}=\int d^{3} x \frac{T}{g^{2}}\left(\nabla \theta_{k}\right)^{2}-\frac{2 T^{3}}{\pi^{2}} \sum_{n=1}^{\infty} \frac{1}{n^{4}}\left(\operatorname{Tr}_{F} P^{n} \operatorname{Tr}_{F} P^{\dagger n}-1\right)+\sum_{k=1}^{[N / 2]} \lambda_{k} \operatorname{Tr} P^{k} \operatorname{Tr} P^{\dagger k}
$$

where the $\theta$ 's are eigenvalues of $P$. In a gauge where $A_{0}$ is time-independent and diagonal we have $P_{j k}=\delta_{j k} \exp \left[i \theta_{j}\right]$. This expression contains a kinetic term obtained by a reduction of the gauge field action as well as an effective potential term obtained from a one-loop calculation of the free energy of the gauge bosons in the presence of a background Polyakov loop. To the standard form of the high-temperature effective action, we have added $V_{\text {ext }}$, which is gauge-invariant and centersymmetric. If all the $\lambda_{k}$ 's are positive, the minimum of $V_{\text {ext }}$ occurs for a unique set of Polyakov loop eigenvalues that are permuted by a $Z(N)$ symmetry transformation [2]. If we denote the corresponding Polyakov loop for an $S U(N)$ model by $P_{0 N}$, then the $Z(N)$ symmetry transformation $P_{0 N} \rightarrow z P_{0 N}$ is equivalent to a gauge transformation $g P_{0 N} g^{\dagger}$. This condition leads immediately to a set of conditions $\operatorname{Tr}_{F} P_{0 N}^{k}=0$ for $k=1$ to $N-1$ consistent with $Z(N)$ symmetry. Thus if $\lambda_{k} \gg T^{3}$ for all $k$ at high temperatures, we can recover the confined phase in a regime where $g(T)$ is small, and perturbation theory is presumably valid. It is only necessary to include terms up to $k=[N / 2]$ because the string tensions in different $N$-alities obey $\sigma_{N-k}=\sigma_{k}$. In contrast, the oneloop perturbative potential terms are minimized by breaking $Z(N)$ completely, with minima for $P \in Z(N)$, corresponding to the deconfined phase. It is the interplay of the different potential terms that leads to a remarkably rich phase structure.

\section{Order Parameters and Phase Diagram for $S U(4)$ and $S U(6)$}

We begin by considering the possible phases of the gauge group $S U(4)$. The center of $S U(4)$ is $Z(4)$, and there is only one non-trivial sub-group, $Z(2)$. A phase in which $Z(4)$ breaks to $Z(2)$ can be characterized by $\left\langle\operatorname{Tr}_{F} P\right\rangle=0$ and $\left\langle\operatorname{Tr}_{F} P^{2}\right\rangle \neq 0$. The other possible phases are the deconfined phase, characterized by $\left\langle\operatorname{Tr}_{F} P\right\rangle \neq 0$ and $\left\langle\operatorname{Tr}_{F} P^{2}\right\rangle \neq 0$, and the confined phase, which has $\left\langle\operatorname{Tr}_{F} P\right\rangle=$ 0 and $\left\langle\operatorname{Tr}_{F} P^{2}\right\rangle=0$. All of the phase transitions between different phases will involve a jump in one or more order parameters, and are first order.

The simplest case to analyze occurs when $\left|\lambda_{k}\right| \gg T^{3}$ for all $k$. In this case, the symmetrybreaking solutions will generally be maximal in the sense that the magnitude $\left|\operatorname{Tr}_{F} P^{k}\right|$ will be $N$, unless it is zero. The phase structure for $S U(4)$, obtained by minimizing $V_{\text {ext }}$ is shown in Fig. 1. The effect of $O\left(T^{3}\right)$ corrections is to shift the location of the tricritical point away from the origin, and accordingly shift the critical lines both horizontally and vertically. This effect is clearly seen in the $S U$ (4) simulations of [1]. Even though $\lambda_{2}=0$, the $Z(2)$ phase was obtained for $\lambda_{1}$ sufficiently positive. In the case of $S U(3)$, the interplay of potential terms when $\lambda_{1}$ is on the order of $T^{3}$ leads to a skewed phase [1].

In the case of $S U(6)$, there are two non-trivial subroups of $Z(6): Z(2)$ and $Z(3)$. In the phase where there is an unbroken $Z(2)$ global symmetry, we have $\left\langle\operatorname{Tr}_{F} P^{2}\right\rangle \neq 0$ and $\left\langle\operatorname{Tr}_{F} P\right\rangle=\left\langle\operatorname{Tr}_{F} P^{3}\right\rangle=$ 0 . If $Z(6)$ breaks to $Z(3)$, we have instead $\left\langle\operatorname{Tr}_{F} P^{3}\right\rangle \neq 0$ and $\left\langle\operatorname{Tr}_{F} P\right\rangle=\left\langle\operatorname{Tr}_{F} P^{2}\right\rangle=0$. The phase structure for $S U(6)$, obtained by minimizing $V_{\text {ext }}$ is shown in Fig. 2 for the case $\lambda_{1}>0$ and in Fig. 3 for the case $\lambda_{1}<0$. Note that the confined and deconfined phases are not directly connected in 


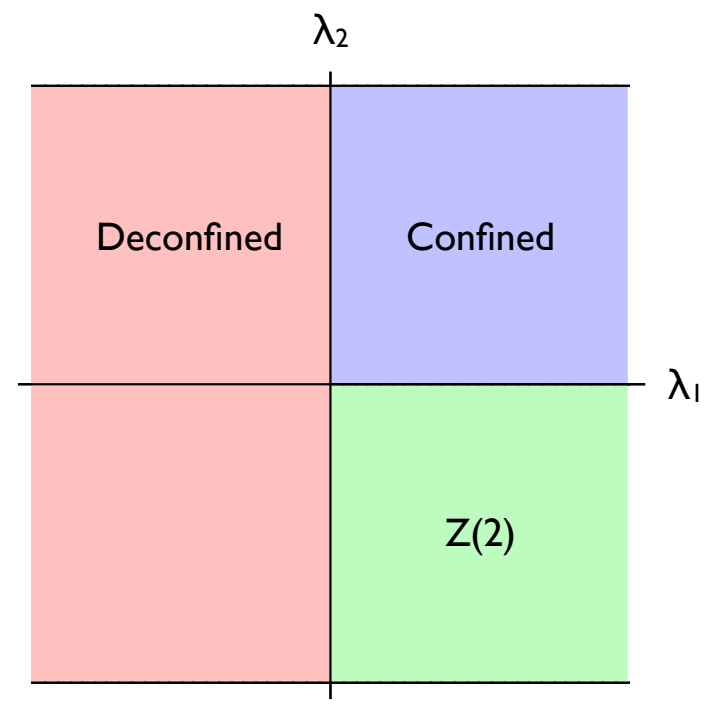

Figure 1: Phase diagram for $S U(4)$.

the case $\lambda_{1}>0$, but are separated by the other phases. As in the case of $S U(4), O\left(T^{3}\right)$ corrections shift the critical lines somewhat, but preserve the overall structure of the phase diagram.

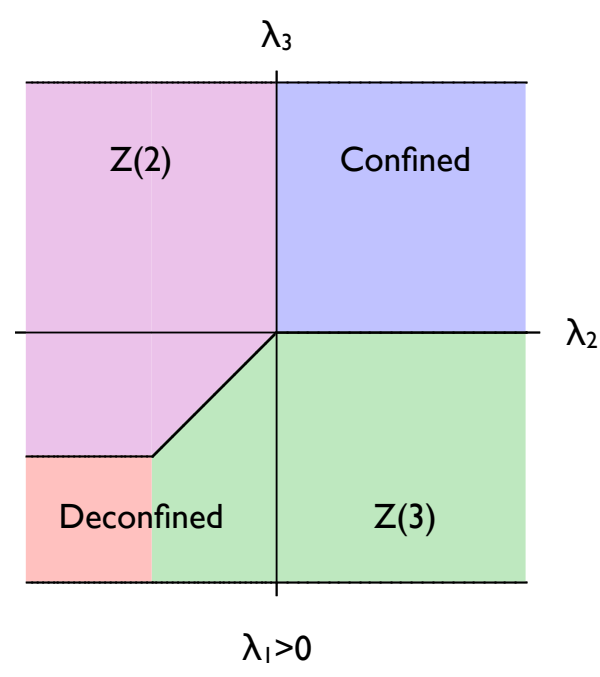

Figure 2: $S U(6)$ phases for $\lambda_{1}>0$.

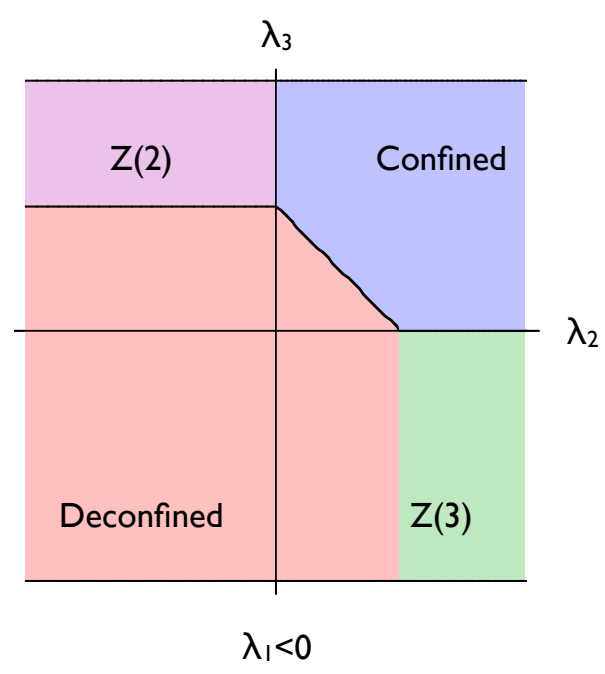

Figure 3: $S U(6)$ phases for $\lambda_{1}<0$.

\section{Properties of Partially Confined Phase}

For an $S U(N)$ gauge theory, we can associate a phase with every integer decomposition of the form $N=L M$, where $Z(L)$ is unbroken, and $Z(M)$ is broken. The value of $P$ at the minimum of the effective potential can be written as an element of $S U(L) \otimes S U(M)$ of the form $P=P_{0 L} \otimes 1_{M}$, where $P_{0 L}$ is associated with the confined phase of $S U(L)$. This $P$ has the property that $\operatorname{Tr}_{F}\left(P_{L}^{(0)}\right)^{k}=0$ 
for all $k=1 . .(L-1)$, and also satisfies $\left(P_{L}^{(0)}\right)^{L}=1_{L}$. The value of the effective potential at the minimum determines the free density and other thermodynamic properties.

String tensions and screening masses are obtained from small fluctuations about the minimum of the effective potential in the usual way. For the sake of simplicity, we describe only typical results here, and generally omit corrections due to the gauge boson term in the effective potential. The simplest cases are the confined and deconfined phases. In the confined phase, the string tensions $\sigma_{k}$ are obtained from

$$
\left\langle\operatorname{Tr} P^{k}(\vec{x}) \operatorname{Tr} P^{\dagger k}(\vec{y})\right\rangle \sim \exp \left[-\frac{1}{T} \sigma_{k}|\vec{x}-\vec{y}|\right]
$$

are given by

$$
\sigma_{k}=k \sqrt{\frac{1}{2} g^{2} N \lambda_{k} T}
$$

It is quite striking that each $\sigma_{k}$ can be varied independently. Of course, in regions where, e.g., $\sigma_{2}>2 \sigma_{1}$, we expect to see that the asymptotic $k=2$ string tension is $2 \sigma_{1}$, due to interactions neglected in the quadratic approximation. In the deconfined phase, the Debye screening mass $m_{D}$ is given by

$$
m_{D}^{2}=\frac{g^{2} N}{T} \sum_{k=1}^{\left[\frac{N}{2}\right]} k^{2} \lambda_{k}
$$

for Polyakov loops of all $\mathrm{N}$-alities.

Partially confined phases have both string tensions and screening masses. In each $k$-sector, the square of the mass is a linear combination of the parameters $\lambda_{k}$. In confined sectors, the string tension $\sigma_{k}$ is the mass divided by $T$. For example, in the case where the $Z(4)$ symmetry of $S U(4)$ is spontaneously broken to $Z(2)$, there is a single string tension for odd values of $k$, and a single screening mass for even values of $k$. The string tension associated with $\operatorname{Tr}_{F} P$ is given by

$$
\sigma_{1}=\sqrt{g^{2} N T\left(\lambda_{1}-4 \lambda_{2}\right)}
$$

and the screening mass associated with $\operatorname{Tr}_{F} P^{2}$ is

$$
m_{D}=2 g \sqrt{-N \lambda_{2} / T}
$$

In a phase where $Z(N)$ is broken to $Z(L), \operatorname{Tr}_{F} P^{L}$ plays a special role. There are $M$ equivalent thermal states associated with the broken $Z(M)$ symmetry. Taking $P=P_{L}^{0} \otimes e^{2 \pi i k / N}$, where $k=$ $0, . .,(M-1)$, we see that $\operatorname{Tr} P^{L}=N \exp [2 \pi i k / M]$. There are one-dimensional kink solutions of the equations of motion derived from the effective action that interpolate between these states. These solutions have a finite action per unit area, which are the 't Hooft loop surface tensions $\rho_{k}$.

The general form of such a kink solution is $P(z)=P_{L}^{0} \otimes e^{i \psi(z)}$. It is easy to calculate $\rho_{k}$ via BPS techniques if one term dominates in the effective potential. For example, in the case where $-\lambda_{L} \gg T^{3}$ and no other $\lambda_{k}$ is negative or too large, we have

$$
\rho_{k}=16(k L)(N-k L) \sqrt{\frac{\left(-\lambda_{L}\right) T}{g^{2} N}} .
$$


This result gives a modified Casimir scaling law:

$$
\frac{\rho_{k}}{\rho_{1}}=\frac{k L(N-k L)}{N-L} .
$$

In general, Casimir scaling will hold whenever the relevant part of the effective potential can be written as a sum of adjoint representation terms, as is the case with our external potential $V_{\text {ext }}$. In the case where the gauge boson contribution to the effective potential dominates in the kink solution, we have

$$
\rho_{k}=\frac{4 \pi^{2}(k L)(N-k L) T^{2}}{3 \sqrt{3 g^{2} N}} .
$$

This requires $T^{3} \gg\left|\lambda_{L}\right|$, as in our $\mathrm{SU}(4)$ simulations, where $\lambda_{2}=0$. This result again obeys Casimir scaling, and represents a generalization for the formula for $\rho_{k}$ in the deconfined phase [15].

\section{Conclusions}

A large number of phases can be created at high temperatures by adding Polyakov loop terms to the action, including many partially confined phases. Phase diagrams can be easily predicted. String tensions can be varied almost arbitrarily, and their values predicted. 't Hooft loop surface tensions can also be calculated, and obey Casimir scaling for a large class of potentials.

The case of $S U(6)$ is particularly interesting. In addition to the confined and deconfined phases, there are two distinct partially confined phases, associated with the $Z(2)$ and $Z(3)$ subgroups of $Z(6)$. This is the natural model to test the analytic predictions we have made for string tensions, screening masses, and other quantities characterizing the different phases.

The original motivation for studying lattice gauge theories with additional couplings to Polyakov loops was to study the variation of string tension scaling behavior. The results of our analytical calculations indicate a class of extended models for which the string tensions can be varied continuously. On the other hand, the surface tensions associated with 't Hooft loops, representing the $Z(N)$ duals of Wilson loops, i.e. magnetic monopole loops, are predicted to obey Casimir scaling in the deconfined and partially confined phases. We are beginning work to confirm these analytical results with lattice simulations. We are also planning a study of the behavior of calorons in these models, which should offer a direct view, both analytically and from simulations, of the role of calorons in confining gauge theories.

\section{References}

[1] J. C. Myers and M. C. Ogilvie, New Phases of SU(3) and SU(4) at Finite Temperature, arXiv:0707.1869 [hep-lat].

[2] P. N. Meisinger, T. R. Miller and M. C. Ogilvie, A phenomenological treatment of chiral symmetry restoration and deconfinement, Nucl. Phys. Proc. Suppl. 119 (2003) 511 [arXiv:hep-lat/0208073].

[3] P. N. Meisinger and M. C. Ogilvie, Non-universality of string tension ratios and gluon confinement at finite temperature, PoS LAT2005 (2006) 195.

[4] P. N. Meisinger and M. C. Ogilvie, String tension scaling in models of the confined phase, PoS LAT2006 (2006) 072 [arXiv:hep-lat/0612002]. 
[5] N. M. Davies, T. J. Hollowood, V. V. Khoze and M. P. Mattis, Gluino condensate and magnetic monopoles in supersymmetric gluodynamics, Nucl. Phys. B 559 (1999) 072 [arXiv:hep-th/9905015].

[6] N. M. Davies, T. J. Hollowood and V. V. Khoze, Monopoles, affine algebras and the gluino condensate, J. Math. Phys. 44 (2003) 3640 [arXiv:hep-th/0006011].

[7] D. Diakonov, N. Gromov, V. Petrov and S. Slizovskiy, Quantum weights of dyons and of instantons with non-trivial holonomy, Phys. Rev. D 70 (2004) 036003 [arXiv:hep-th/0404042].

[8] P. N. Meisinger and M. C. Ogilvie, Complete high temperature expansions for one-loop finite temperature effects, Phys. Rev. D 65 (2002) 056013 [arXiv:hep-ph/0108026].

[9] D. J. Gross, R. D. Pisarski and L. G. Yaffe, QCD And Instantons At Finite Temperature, Rev. Mod. Phys. 53 (1981) 43.

[10] N. Weiss, The Effective Potential For The Order Parameter Of Gauge Theories At Finite Temperature, Phys. Rev. D 24 (1981) 475.

[11] T. Bhattacharya, A. Gocksch, C. Korthals Altes and R. D. Pisarski, Interface tension in an $S U(N)$ gauge theory at high temperature, Phys. Rev. Lett. 66 (1991) 998.

[12] T. Bhattacharya, A. Gocksch, C. Korthals Altes and R. D. Pisarski, Z(N) interface tension in a hot SU(N) gauge theory, Nucl. Phys. B 383 (1992) 497 [arXiv:hep-ph/9205231].

[13] F. Bursa and M. Teper, Casimir scaling of domain wall tensions in the deconfined phase of $D=3+1$ $S U(N)$ gauge theories, JHEP 0508 (2005) 060 [arXiv:hep-lat/0505025].

[14] P. de Forcrand, B. Lucini and D. Noth, 't Hooft loops and perturbation theory, PoS LAT2005 (2006) 323 [arXiv:hep-lat/0510081].

[15] P. Giovannangeli and C. P. Korthals Altes, 't Hooft and Wilson loop ratios in the QCD plasma, Nucl. Phys. B 608 (2001) 203 [arXiv:hep-ph/0102022]. 